\title{
Nonempirical Calculations on Excited States: The Ethylene Molecule
}

\author{
Thom. H. Dunning* and Vincent McKoy \\ Gates and Crellin Laboratories of Chemistry, $\uparrow$ California Institute of Technology, Pasadena, Calıfornia
}

(Received 2 March 1967)

\begin{abstract}
A series of nonempirical calculations are reported on the excited states of the ethylene molecule using a recent minimum basis set LCAO MO SCF wavefunction. For the lowest excited singlet state of ethylene $\left({ }^{1} B_{3 u}\right)$ the coupling between the $\pi$ electrons and $\sigma$ electrons is significant: the excitation energy being decreased from 11.98 to $10.17 \mathrm{eV}$ and the oscillator strength from 1.03 to 0.73 . This coupling has little effect on the triplet state. In the next higher approximation (the random-phase approximation) the excitation energy is further decreased to $9.44 \mathrm{eV}$ and the transition moment to 0.51 . With the use of accurate LCAO MO SCF wavefunctions, it is felt that the methods presented here will provide a basis for the theoretical interpretation of electronic spectra.
\end{abstract}

\section{INTRODUCTION}

\section{A. General}

Now that the application of the Hartree-FockRoothaan treatment of the electronic structure of molecules is practical for many molecules of chemical interest, ${ }^{1-3}$ one of the more important problems in molecular quantum mechanics is assessing the effect of electronic correlation particularly for observables other than the total energy. To obtain even a simple approximate Hartree-Fock wavefunction for a relatively small molecule requires a fair amount of effort and computer time. Very accurate Hartree-Fock wavefunctions for the ground state would demand a prohibitively large investment. If one is interested in properties related to two states, the problem becomes even more formidable, and correlation effects have still not been included. What we show in this paper is that correlation effects are significant in locating an important excited state of ethylene (for both excitation energy and oscillator strength for the singlet state located at $7.6 \mathrm{eV}$ experimentally), but that it can be described quite economically from a simple ground-state Hartree-Fock calculation by applying, among others, the theory of the random-phase approximation (RPA) for electronic correlation. We chose the methods for their conceptual usefulness and potential for future applications, but they are certainly not the only ones which would yield these results.

The methods to be employed have been widely used both in nuclear and solid-state physics ${ }^{4-7}$ and more recently in the study of electronic correlation in atoms ${ }^{8}$

\footnotetext{
* Woodrow Wilson Foundation Predoctoral Fellow 1965-1966. † Contribution No. 3527.

${ }^{1}$ L. C. Allen and A. M. Karo, Rev. Mod. Phys 32, 275 (1960).

2 B. J. Ransil, Rev. Mod. Phys. 32, 245 (1960).

a R. M. Pitzer and W. N. Lipscomb, J. Chem Phys. 39, 1995 (1963); W E, Palke and W. N. Lipscomb, J. Am. Chem. Soc. 88, 2384 (1966).

4 J. P. Elliott and B. H Flowers, Proc. Roy Soc. (London) A242, 57 (1957).

D D. J. Thouless, Nucl. Phys. 22, 78 (1961)

6 P. W. Anderson, Phys. Rev. 112, 1900 (1958).

7 H. Suhl and N. R. Werthamer, Phys. Rev. 122, 359 (1961).

8 P. L. Altick and A. E. Glassgold, Phys Rev 133, 632 (1964).
}

and molecules. ${ }^{9}$ The theory as applied here is not very difficult and the physical concepts are simple. In keeping with the usual procedure in these fields, we derive the equations using the formalism of second quantization. In this representation we can make reasonable approximations which are difficult to formulate in the coordinate representation. All of the equations derived in this paper are obtainable from appropriate variational procedures, but the method employed here has the advantage that the approximations are explicitly displayed and the removal of any difficulties is a conceptually straightforward matter.

We chose ethylene as our example as its spectrum is of considerable interest. It is not necessary to stress the importance of understanding the ethylene molecule quantum mechanically. It is the simplest $\pi$-electron system and a prototype for larger $\pi$-electron molecules. If we want to know the properties of some of its excited states, we need to have some physical idea as to how "to get" this state from the ground state. The problem is one of finding a form for an operator $A^{+}(E)$ such that it satisfies the following equation:

$$
\left[\mathfrak{F C}, A^{+}(E)\right]|0\rangle=\Delta E A^{+}(E)|0\rangle,
$$

where $\mathfrak{H}$ is the complete electronic Hamiltonian for the molecular system under consideration. The operator $A^{+}(E)$ contains whatever physical information we have about the excited state of the system. We see that the operator $A^{+}(E)$ generates an excited state of the Hamiltonian $\mathfrak{H}$, with excitation energy $\Delta E$, when it operates on the ground state; i.e.,

$$
A^{+}(E)|0\rangle=|E\rangle \text {. }
$$

Note that the above describes a vertical excitation. In practice, because of electronic interaction, $A^{+}(E)$ cannot be determined such that the above equation is exactly satisfied, but rather we have

$$
\begin{aligned}
{\left[\mathfrak{H}, S^{+}(E)\right] } & =\Delta E S^{+}(E)+R \\
& \approx \Delta E S^{+}(E),
\end{aligned}
$$

${ }^{9}$ A. Herzenberg, D. Sherrington, and M. Suveges, Proc. Phys, Soc. (London) 84, 465 (1964). 


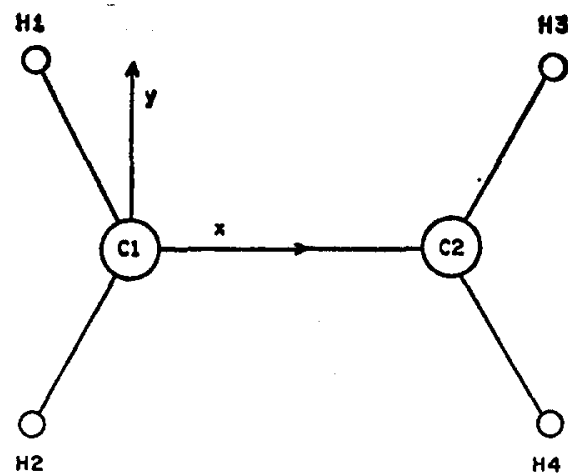

FIG. 1. The geometry of ethylene.

where $R$ represents all the terms which cannot be reduced into a form consistent with $S^{+}(E)$. The operator $S^{+}(E)$ generates an approximate excited state of the system when it acts on the ground state. It is one of the variables of the problem in whose formulation we may utilize our chemical intuition.

In this paper we consider the following three approximations for the excited states created by the excitation operator $S^{+}(E)$.

(1) The single-transition approximation (STA $)^{10}$ is the approximation most frequently used by chemists. It assumes that the excited state can be represented by a single particle above the set of levels occupied in the ground state coupled with a hole within that set, e.g., the ${ }^{1} B_{3 u} \leftarrow^{1} A_{1 g}$ transition in ethylene would correspond to an electron going from a $\pi$-bonding to a $\pi$-antibonding orbital.

(2) In the Tamm-Dancoff approximation (TDA) 4,9 the excited state is represented as a linear combination of single-particle transitions. This method is encountered quite frequently in molecular quantum mechanics, e.g., in the study of the spectra of aromatic molecules by Pople. ${ }^{11}$ Again, with ethylene as an example, we now include in the excited-state wavefunction configurations corresponding to moving an electron from the " $\sigma$ " and " $\mathrm{CH}$ " bonding orbitals (the $\sigma$ core) into antibonding levels.

(3) In the random-phase approximation (RPA $)^{6,8}$ the excited state is still represented by a linear combination of single-particle transitions except that now we allow the ground state to include configurations other than just the Hartree-Fock (HF) component, i.e., the effects of configuration interaction (CI) are, to some extent, taken into account. We still solve an eigenvalue equation for $\Delta E$ directly. This, as expected, is an improvement on Method (2).

In the above a "hole" state corresponds to an unoccupied level within the normal HF ground state while a "particle" state denotes an occupied virtual orbital. Excitation of the type considered here creates

10 C. C. J. Roothaan, Rev. Mod. Phys. 23, 69 (1951).

11 J. A. Pople, Proc. Phys. Soc. (London) A68, 81 (1955). "particle-hole" pairs, each such pair having its own frequency (excitation energy). By diagonalizing the Hamiltonian matrix within this set we find the normal modes of the assembly of coupled oscillators. Surprisingly, such coupling is not negligible in ethylene as it reduces the oscillator strength given by the STA by a factor of 2 .

\section{B. The Ethylene Molecule}

To provide a concrete foundation for the above approximations, we have done the complete set of calculations on the ethylene molecule utilizing the recent minimum basis set LCAO MO SCF calculation by Palke and Lipscomb. ${ }^{12}$ The excited state in which we are most interested is the one which arises from the so-called $\pi \rightarrow \pi^{*}$ transitions which, if we define the coordinate system as shown in Fig. 1, is of symmetry $B_{3 u}$ with the emitted radiation polarized along the $x$ axis. Since some new concepts have arisen from this work, especially in regard to the $\pi$-electron approximation, we briefly review three of the more significant $\pi$-electron calculations on ethylene in order to gain the proper perspective.

The first nonempirical $\pi$-electron calculation on ethylene was by Parr and Crawford ${ }^{13}$ using the formalism developed by Goeppert-Mayer and Sklar. ${ }^{14}$ Their results are typical of nonempirical $\pi$-electron calculations: the predicted spectra being in qualitative, but not quantitative, agreement with experiment. The results of this calculation, as well as the following two, are listed in Table I along with the experimentally observed excitation energies and oscillator strengths. Still within the $\pi$-electron approximation, Murai ${ }^{15}$ relaxed the constraints on the $\pi$-molecular orbitals by allowing the orbital exponents of the atomic Slater orbitals to be a function of the state of the system. As is evident from Table $I$, the triplet state is now in better agreement with experiment while the singlet

TABLE I. $\pi$-Electron calculations on the excited $B_{3 u}$ state of ethylene: excitation energies (in electron volts), oscillator strengths, and ionization potential."

\begin{tabular}{lrrrr}
\hline & $\begin{array}{c}\text { Parr and } \\
\text { Crawford }\end{array}$ & Murai & Huzinaga & Exptl \\
\hline${ }^{8} B_{3 u}(T)$ & 3.10 & 4.60 & 4.45 & 4.6 \\
${ }^{1} B_{3 u}(V)$ & 11.50 & 11.20 & 7.28 & 7.5 \\
$f\left({ }^{1} B_{8 u}\right)$ & & & & $\sim 0.3^{\mathrm{b}}$ \\
I.P. & 11.90 & 8.80 & 8.52 & 10.52 \\
\hline
\end{tabular}

- Except as noted. see text Ref. 28.

b M. Zelikoff and K. Watanabe, J. Opt. Soc. Am. 43, 756 (1953).

12 W. E. Palke and W. N. Lipscomb (private communication). ${ }^{13}$ R. G. Parr and B. L. Crawford, J. Chem. Phys. 16, 526 (1948).

${ }_{14}$ M. Goeppert-Mayer and A. L. Sklar, J. Chem. Phys. 6, 645 (1938).

${ }^{16}$ T. Murai, Progr. Theoret. Phys. (Kyoto), 7, 345 (1952). 
state, which was in much need of improvement, has hardly changed. Finally, Huzinaga ${ }^{16}$ carried the $\pi$ electron approximation to its fullest by permitting the bonding and antibonding $\pi$-molecular orbitals to have different exponents as well as allowing the exponents to vary with the state. The predicted spectra now agree very well with experiment. However, the calculated ionization potential is far below the experimental.

The $\pi$-electron treatment of ethylene has attained a refinement which cannot be approached in larger $\pi$ electron systems and still all the experimental facts cannot be adequately explained. An improvement in the theoretically calculated value of one observable is attained only at the expense of poor values for other observables. We feel that the calculations presented in this paper go a long way towards resolving these diffculties. We can see the role played by electronic correlation in the excitation process and discuss a simple method of taking it into account.

One may have expected that the problem of the spectra of ethylene would be resolved once an accurate 16-electron Hartree-Fock treatment of the molecule was available, for then the core potential would be adequately defined. However, the locations of the excited states obtained from Palke and Lipscomb's LCAO MO SCF wavefunction are comparable to those obtained by $\pi$-electron theory (compare the STA results in Tables $\mathrm{V}$ and VI with those for the Parr and Crawford calculation given in Table I) and therefore the trouble with predicting the spectra does not arise completely from the $\pi$-electron approximation for the ground-state calculation.

The source of the trouble is the neglect of the change in the $\sigma$ core upon excitation; the $\pi$ electrons being coupled by the residual electronic interaction to the $\sigma$ electrons. In fact, this coupling is sufficiently strong to cause a decrease of about $2 \mathrm{eV}$ in the excitation energy and a decrease of $30 \%$ in the oscillator strength for the ${ }^{1} B_{3 u} \leftarrow{ }^{1} A_{10}$ transition compared to the $\pi \rightarrow \pi^{*}$ approximation. As expected, the RPA further decreases the excitation energy to $9.44 \mathrm{eV}$ and the oscillator strength to 0.51 .

A word of caution is appropriate at this point. Our calculations are based on a minimum basis set LCAO MO SCF calculation and as such we have not reached the Hartree-Fock molecular-orbital limit. As a remedy for this we could enlarge the basis set, for example, see the results of Moskowitz and Harrison ${ }^{17}$ using a large set of Gaussian orbitals which predict values for the excitation energies using the $\pi \rightarrow \pi^{*}$ approximation which are almost as good as ours using the RPA.

The methods discussed here are not the only ones relevant to the problem. One could use the LCAO MO SCF results as a basis for an extensive configuration-

16 S. Huzinaga, J. Chem. Phys. 36, 453 (1962).

${ }_{17} \mathrm{~J}$. W. Moskowitz and M. C. Harrison, J. Chem. Phys. 42, 1726 (1965) ; J. S. Schulman, J. W. Moskowitz, and C. Hollister, J. Chem. Phys. 46, 2759 (1967). interaction calculation on both the ground state and excited states. Besides being uneconomical (for a large number of molecular integrals must be assembled), the following consideration must be taken into account. In the usual CI calculation one mixes in components corresponding to double excitations from electrons in the same spatial orbital. These are usually the most important. However, the lowering that we observe while essentially being a correlation effect is due to correlations between electrons in orbitals that are quite different spatially, i.e., as Herzenberg et al. ${ }^{9}$ state "correlations extending from bond to bond."

Alternately, one could do an open-shell LCAO MO SCF calculation on the excited states with the hope that correlation effects would cancel. This method would allow for some relaxation of the $\sigma$ electrons and should give an excitation energy lower than the STA. However, preliminary open-shell calculations in this Laboratory and others (e.g., see Ref. 17) indicate that the relaxation of the core electrons has a small effect on the excitation energy and the oscillator strength. These results emphasize that the important coupling is due to the residual $\sigma-\pi$ interaction, i.e., it is a correlation effect.

As a brief outline of the remainder of the paper, in Sec. II we discuss the main ideas of second quantization and then derive the equations related to the various approximations to $A^{+}(E)$. In Sec. III the results of the calculations on ethylene are presented in detail. In Sec. IV we discuss the results and comment on their interpretation.

\section{THEORY}

The electronic Hamiltonian for the molecular system is, in atomic units,

$$
\begin{aligned}
\mathfrak{H} & =\sum_{i}\left[-\frac{1}{2} \nabla_{i}{ }^{2}-\left(\sum_{\alpha} Z_{\alpha} / r_{\alpha i}\right)\right]+\frac{1}{2} \sum_{i} \sum_{j} r_{i j}{ }^{-1} \\
& =\sum_{i} B_{i}+\frac{1}{2} \sum_{i} \sum_{j} v_{i j} .
\end{aligned}
$$

In the Hartree-Fock approximation we replace the electronic interaction term, $\frac{1}{2} \sum_{j} v_{i j}$, by an effective one-particle potential, $V_{i}$. The lowest $N$ eigenfunctions of the new Hamiltonian

$$
(H+V)|i\rangle=\epsilon_{i}|i\rangle
$$

are then combined into a Slater determinant in order to form the Hartree-Fock molecular wavefunction, $|\mathrm{HF}\rangle$.

For molecular systems explicit numerical solution of the Hartree-Fock equations is intractable and an additional approximation is required; namely, that the molecular orbital, $|i\rangle$, can be satisfactorily approximated by a linear combination of atomic orbitals centered on the various nuclei (the LCAO approximation). The Hartree-Fock, or molecular-orbital, limit can, thus, be achieved only through the use of large basis sets. 
Combining Eqs. (4b) and (5) the complete electronic Hamiltonian can be written as

$$
\mathfrak{H}=\sum_{i}\left(H_{i}+V_{i}\right)+\sum_{i}\left(\frac{1}{2} \sum_{j} v_{i j}-V_{i}\right) .
$$

The last two terms comprise the residual interaction and give rise to electronic correlation.

In the notation of second quantization ${ }^{18}$ the Hamiltonian is

$$
\begin{aligned}
\mathfrak{F}=\sum_{i} \epsilon_{i} a_{i}{ }^{+} a_{i}+\frac{1}{2} \sum_{i j k l} \mathrm{~V}_{i j k l} a_{j}{ }^{+} a_{i}+a_{k} a_{l} \\
-\sum_{j k \beta}\left(\mathbf{V}_{\beta j \beta k}-\mathbf{V}_{\beta j k \beta}\right) a_{j}{ }^{+} a_{k},
\end{aligned}
$$

where we have introduced the explicit form of the Hartree-Fock one-particle potential, $V_{i}$. The operators $a_{i}{ }^{+}$and $a_{i}$ are the creation and annihilation operators. The operator $a_{i}^{+}$acting on a state puts an electron into level $i$ while $a_{i}$ does just the reverse. The usefulness of this "occupation number" representation is in part due to the fact that in this new representation we can make reasonable approximations which are very difficult to formulate in the coordinate representation.

The integrals $\mathrm{V}_{i j k l}$ are defined by

$$
\mathrm{V}_{i j k l}=\iint \phi_{i}{ }^{*}(1) \phi_{j}^{*}(2) r_{12}^{-1} \phi_{k}(1) \phi_{l}(2) d v_{1} d v_{2},
$$

the $\phi_{i}$ 's denoting the molecular spin orbitals. In this equation and the ones to follow, we denote by the subscripts

$$
\begin{aligned}
\alpha, \beta, \gamma, \delta, \cdots & \text { single-particle states occupied in the } \\
& \text { Hartree-Fock ground state (i.e., hole } \\
& \text { states), }
\end{aligned}
$$

If we define the Fermi level, $\epsilon_{F}$ (we use it just for vocabulary purposes), as being the uppermost level which is occupied in the Hartree-Fock ground state, then in the Hartree-Fock approximation the orbital occupation numbers are given by

$$
\begin{array}{ll}
n_{i}=1, & \epsilon_{i} \leq \epsilon_{F} ; \\
n_{i}=0, & \epsilon_{i}>\epsilon_{F} .
\end{array}
$$

These are just the expectation values of the number operators $\hat{n}_{i}=a_{i}{ }^{+} a_{i}$ over the HF ground state. A transition will then be defined as removing an electron from below the Fermi level and placing it above.

We now need the precise form of the excitation operator, $S^{+}(E)$, in second quantization. But, before doing this we require a further property of the exact

${ }^{18}$ A concise discussion of the formalism of second quantization may be found in L. D. Landau and E. M. Lifshitz, Quantum Mechanics, Non-Relativistic Theory (Pergamon Press Ltd., London, 1958), pp. 215-223. excitation operator. Denoting the eigenstates of the Hamiltonian $B$ by $|0\rangle,|E\rangle$, etc., the equations defining the excitation operator $A^{+}(E)$ are just Eqs. (1a) and (1b). Taking the Hermitian conjugate of Eq. (1), we can derive a relationship connecting the true ground state and the Hermitian conjugate of the excitation operator, namely,

$$
A(E)|0\rangle=0 .
$$

This property of $A(E)$ will be used frequently and places restrictions on the choice of the approximate excitation operator, $S^{+}(E)$; i.e., given an approximate $|0\rangle, S^{+}(E)$ must be such that its Hermitian conjugate satisfies the above equation and vice versa.

Let us now define a particle-hole creation operator

$$
C^{+}(m \alpha)=a_{m}{ }^{+} a_{\alpha}
$$

and the corresponding destruction operator

$$
C(m \alpha)=a_{\alpha}{ }^{+} a_{m} .
$$

These operators will be the basic building blocks used to construct the various approximations to the excitedstate operator; for example,

$$
C^{+}(m \alpha)|\mathrm{HF}\rangle=\left|\begin{array}{l}
m \\
\alpha
\end{array}\right\rangle
$$

a Slater determinant derived from the HF ground state by replacing the orbital $|\alpha\rangle$ by the virtual orbital $|m\rangle$. With these definitions, the particle-hole creation and destruction operators satisfy the following commutation relations:

$$
\begin{aligned}
{\left[C^{+}(m \alpha), C^{+}(n \beta)\right] } & =[C(m \alpha), C(n \beta)]=0, \\
{\left[C(m \alpha), C^{+}(n \beta)\right] } & =\delta_{m n} \delta_{\alpha \beta}-\delta_{\alpha \beta} a_{n}{ }^{+} a_{m}-\delta_{m n} a_{\beta}{ }^{+} a_{\alpha},
\end{aligned}
$$

whereas the operators $a_{i}^{+}$and $a_{i}$ satisfy the anticommutation relation

$$
\begin{gathered}
\left\{a_{i}^{+}, a_{j}^{+}\right\}=\left\{a_{i}, a_{j}\right\}=0, \\
\left\{a_{i}+, a_{j}\right\}=\delta_{i j} .
\end{gathered}
$$

The eigenvalue equations for the various forms of $S^{+}(E)$ can all be derived from a consideration of the equation $^{19}$

$$
\left\langle E\left|(E-\mathfrak{H}) C^{+}(m \alpha)\right| 0\right\rangle=0,
$$

which after a little manipulation becomes

$\left(E-E_{0}\right)\left\langle E\left|C^{+}(m \alpha)\right| 0\right\rangle-\left\langle E\left|\left[\mathfrak{H}, C^{+}(m \alpha)\right]\right| 0\right\rangle=0$.

Using the property of the excitation operator, Eq. (1), and its Hermitian conjugate, Eq. (8), the above can

${ }^{19}$ The derivation given here follows closely that of Altick and Glassgold, Ref. 8. 


\section{be rewritten as}

$$
\begin{aligned}
\left(E-E_{0}\right)\langle 0| & {\left[A(E), C^{+}(m \alpha)\right]|0\rangle } \\
& -\left\langle 0\left|\left\{A(E),\left[\mathfrak{H C}, C^{+}(m \alpha)\right]\right\}\right| 0\right\rangle=0 .
\end{aligned}
$$

Note the way in which the pertinent eigenvalues appear, as $\Delta E=E-E_{0}$. One need only postulate an approximate excitation operator and a consistent ground state; the above then provides the necessary equations for calculating the wavefunction and excitation energy of the excited state.

From Eq. (14) we see that all of the subsequent derivations will have one thing in common-the commutator $\left[\mathcal{H}, C^{+}(m \alpha)\right]$, which is independent of our choice of $S^{+}(E)$. Using the second quantized form of the Hamiltonian, we find that

$\left[\Re \mathcal{K}, C^{+}(m \alpha)\right]=\left(\epsilon_{m}-\epsilon_{\alpha}+\mathrm{V}_{m \alpha \alpha m}-\mathbf{V}_{m \alpha m \alpha}\right) C^{+}(m \alpha)+\sum_{(n \beta)}^{\prime}\left(\mathbf{V}_{\alpha n m \beta}-\mathbf{V}_{\alpha n \beta m}\right) C^{+}(n \beta)+\sum_{(n \beta)}\left(\mathbf{V}_{\alpha \beta m n}-\mathbf{V}_{\alpha \beta n m}\right) C(n \beta)+Q$,

where ${ }^{20}$

$$
\begin{aligned}
Q= & \sum_{(\delta, \gamma)}\left(\mathrm{V}_{\alpha \delta m \gamma}-\mathrm{V}_{\alpha \delta \gamma m}\right) C^{+}(\delta \gamma)+\sum_{(p, r)}\left(\mathrm{V}_{\alpha p m r}-\mathrm{V}_{\alpha p r m}\right) C^{+}(p r) \\
& -\frac{1}{2} \sum_{i j k}\left(\mathrm{~V}_{i j k m}-\mathrm{V}_{i j m k}\right) a_{\alpha} a_{j}^{+} a_{i}{ }^{+} a_{k}+\frac{1}{2} \sum_{j k l}\left(\mathrm{~V}_{\alpha j k l}-\mathrm{V}_{j \alpha k l}\right) a_{m}{ }^{+} a_{j}{ }^{+} a_{k} a_{l} \\
& -\sum_{(\beta \delta)}\left(\mathrm{V}_{\beta \delta \beta m}-\mathrm{V}_{\beta \delta m \beta}\right) C^{+}(\delta \alpha)-\sum_{(\beta n)}\left(\mathrm{V}_{\beta n \beta m}-\mathrm{V}_{\beta n m \beta}\right) C^{+}(n \alpha) \\
& +\sum_{(\beta \delta)}\left(\mathrm{V}_{\beta \alpha \beta \delta}-\mathrm{V}_{\beta \alpha \delta \beta}\right) C^{+}(m \delta)+\sum_{(\beta n)}\left(\mathrm{V}_{\beta \alpha \beta n}-\mathrm{V}_{\beta \alpha n \beta}\right) C^{+}(m n)
\end{aligned}
$$

This splits the commutator into two groups: the first group contains all the single particle-hole terms while $Q$ contains no net single particle-hole terms but contains interactions which might be described as hole-hole $\left[C^{+}(\delta \gamma)\right]$, particle-particle $\left[C^{+}(p r)\right]$, multiple particlehole $\left[C^{+}(n \beta) C^{+}(p \gamma)\right]$, etc. In this paper we are only interested in excited states which can adequately be represented by an elementary transition or a linear combination of elementary transitions. Thus, in the above commutator only single particle-hole interactions are retained. We may now neglect $Q$ and linearize the commutator to

$$
\begin{array}{r}
{\left[\mathfrak{F C}, C^{+}(m \alpha)\right] \approx\left(\epsilon_{m}-\epsilon_{\alpha}+\mathrm{V}_{m \alpha \alpha m}-\mathrm{V}_{m \alpha m \alpha}\right) C^{+}(m \alpha)} \\
+\sum_{(n \beta)}^{\prime}\left(\mathrm{V}_{\alpha n m \beta}-\mathrm{V}_{\alpha n \beta m}\right) C^{+}(n \beta) \\
+\sum_{(n \beta)}^{\prime}\left(\mathrm{V}_{\alpha \beta m n}-\mathrm{V}_{\alpha \beta m n}\right) C(n \beta) .
\end{array}
$$

Note that (1) the above derivation has ignored spin, the resulting equations can be specialized for singlets and triplets in a final step and (2) the prime on the summations indicate exclusion of the term $(m \alpha)$.

In second quantization the dipole moment operator is

$$
\mathbf{r}=\sum_{i, j} \mathrm{~d}_{i j} a_{i}{ }^{+} a_{j}
$$

where

$$
\mathbf{d}_{i j}=\langle i|\mathbf{r}| j\rangle=\int \phi_{i}{ }^{*} \mathbf{r} \phi_{j} d v
$$

${ }^{20}$ As given, it appears that $Q$ contains single particle-hole interactions. However, when the commutator $[A(E), Q]$ is evaluated over the HF ground state, these particle-hole interactions are canceled by corresponding ones in the third and fourth terms of Eq. (16). Equation (16) was retained in its present form for convenience.
Again, retaining only the particle-hole interactions, this reduces to

$$
\mathrm{r} \approx \sum_{(m \alpha)} \mathrm{d}_{m \alpha}\left[C^{+}(m \alpha)+C(m \alpha)\right]
$$

The dipole transition moment is defined as

$$
\mathrm{D}=-\langle E|\mathbf{r}| 0\rangle
$$

or, using Eqs. (2) and (8),

$$
\mathrm{D}=-\langle 0|[A(E), r]| 0\rangle \text {. }
$$

We are now in a position to derive the equations relating to the various approximations to the excitedstate operator.

\section{A. The Single-Transition Approximation ${ }^{10}$}

In this approximation, the one most commonly used by chemists, the excited-state operator is represented by a single particle-hole creation operator, i.e.,

$$
S^{+}(E)=C^{+}(m \alpha) \text {. }
$$

Thus, we speak of $\pi \rightarrow \pi^{*}$ transitions, $n \rightarrow \pi^{*}$ transitions, etc. However, this method, frequently called the frozen core approximation is too rigid; it constrains the orbitals to retain their ground-state form. It is hardly likely that the molecular orbitals which are consistent for the excited state are the same as those for the ground state. ${ }^{21}$

Using Eq. (17) and the commutators Eqs. (10a) and (10b), we find that Eq. (14) becomes

$$
E-E_{0}=\epsilon_{m}-\epsilon_{\alpha}+\mathrm{V}_{m \alpha \alpha m}-\mathrm{V}_{m \alpha m \alpha}
$$

21 S. R. LaPaglia and O. Sinanoğlu, J. Chem. Phys. 44, 1888 (1966). 
Making the excited states eigenfunctions of spin, we get for singlets

$$
\begin{aligned}
{ }^{1} E-E_{0} & =\epsilon_{m}-\epsilon_{\alpha}+2 V_{m \alpha \alpha m}-V_{m \alpha m \alpha} \\
& =\epsilon_{m}-\epsilon_{\alpha}+2 K_{m \alpha}-J_{m \alpha}
\end{aligned}
$$

and for triplets

$$
{ }^{3} E-E_{0}=\epsilon_{m}-\epsilon_{\alpha}-J_{m \alpha},
$$

which are the equations derived by Roothaan in $1951 .{ }^{10}$ Note that the $V_{i j k l}$ are defined in terms of molecular orbitals.

Using Eqs. (18) and (19) the dipole transition moment is

$$
\begin{aligned}
& { }^{1} \mathrm{D}_{m \alpha}=-\sqrt{2}\langle m|\mathrm{r}| \alpha\rangle=-\sqrt{2} \mathrm{~d}_{m \alpha}, \\
& { }^{3} \mathrm{D}_{m \alpha}=0
\end{aligned}
$$

where the superscript denotes the spin multiplicity.

In the derivation of the excitation energies and transition moments for the singlet and triplet states, we have assumed that the molecular orbitals involved are nondegenerate, which is the case for ethylene. If this is not true, say for acetylene, then the appropriate modifications must be made. In any case Eq. (19) is the starting point.

\section{B. The Tamm-Dancoff Approximation ${ }^{4,9}$}

In this approximation we assume that the excited state can be adequately represented by some linear combination of single-particle excitations of the appropriate symmetry. The net effect of this is to allow, in some restricted way, for the rearrangement of the total charge density during the excitation. On the basis of chemical intuition we would expect that the above linear combination would have a major component which defines the essentials of the excitation. This allows us to retain in a limited sense the classification of transitions as being $\pi \rightarrow \pi^{*}$ etc., but this is certainly not required. The effect of the minor components on the excitation energy and transition moment is a matter which can best be settled by numerical calculation, although they had previously been assumed small unless degeneracy was involved. Certainly on the basis of the one-electron levels one might not have expected these minor components to have the effect we soon find. It is the coupling together of many of these excitations that leads to a modification of the lowest-lying state of ethylene.

The excitation operator is

$$
S^{+}(E)=\sum_{(m \alpha)} g(m \alpha ; E) C^{+}(m \alpha) .
$$

Use of Eqs. (17) and (14) and the commutator relations for the particle-hole operators gives the eigenvalue equation to be satisfied by the particle-hole amplitudes, $g(m \alpha ; E)$,

$$
\begin{aligned}
& {\left[\epsilon_{m}-\epsilon_{\alpha}+\mathrm{V}_{m \alpha \alpha m}+\mathrm{V}_{m \alpha m \alpha}-\left(E-E_{0}\right)\right] g(m \alpha ; E)} \\
& +\sum_{(n \beta)}\left(\mathbf{V}_{\alpha n m \beta}-\mathbf{V}_{\alpha n \beta m}\right) g(n \beta ; E)=0 .
\end{aligned}
$$

For convenience the designation " $E$ " in the particlehole amplitudes is dropped. Specializing the above to describe eigenfunctions of spin as before, we get

for singlets:

$$
\begin{aligned}
& {\left[\epsilon_{m}-\epsilon_{\alpha}+2 V_{m \alpha \alpha m}-V_{m \alpha m \alpha}-\left({ }^{1} E-E_{0}\right)\right] g(m \alpha)} \\
& +\sum_{(n \beta)}^{\prime}\left(2 V_{\alpha n m \beta}-V_{\alpha n \beta m}\right) g(n \beta)=0
\end{aligned}
$$

and for triplets:

$$
\begin{aligned}
{\left[\epsilon_{m}-\epsilon_{\alpha}-V_{m \alpha m \alpha}-\left({ }^{3} E-E_{0}\right)\right] g(m \alpha) } & \\
& -\sum_{(n \beta)}^{\prime}\left(V_{\alpha n \beta m}\right) g(n \beta)=0 .
\end{aligned}
$$

These are just the equations derived by Herzenberg et $a l^{9}$ in their semiempirical calculation, including the $\sigma$ electrons, on the spectra of ethylene.

The transition moment in this approximation is

$$
{ }^{1} \mathrm{D}_{\mathrm{TDA}}=\sqrt{2} \sum_{(m \alpha)} g(m \alpha) \mathrm{d}_{m \alpha} .
$$

These results could also have been obtained by a linear variational procedure. If we represent the excited state by

$$
|E\rangle=\sum_{(m \alpha)} g(m \alpha)\left|\begin{array}{l}
m \\
\alpha
\end{array}\right\rangle
$$

and apply the variational principle to determine the amplitudes $g(m \alpha)$, Eq. (24) would result.

\section{The Random-Phase Approximation ${ }^{5,8}$}

Using the language of $\mathrm{CI}$, the ground-state wavefunction to first order is represented by the expansion

$$
|0\rangle=C_{0}|\mathrm{HF}\rangle+\sum_{(m \alpha)} \sum_{(n \beta)} C_{\alpha \beta}^{m n}\left|\begin{array}{l}
m n \\
\alpha \beta
\end{array}\right\rangle,
$$

where

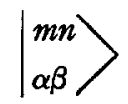

is a double excitation from the orbitals occupied in the HF ground state $(\alpha \beta)$ to the virtual orbitals $(m n)$. We still represent the excited state as being a linear combination of single-particle transitions except that now, using the above ground state, a single-particle transition may be "obtained" in two ways: (1) by exciting from the $\mathrm{HF}$ ground state [the associated operator being $\left.C^{+}(m \alpha)\right]$ or (2) by de-exciting from one of the doubly excited components of the true ground state [the associated operator being $C(n \beta)]$. In view of this 
we would expect that a better approximation to the excitation operator would be

$$
S^{+}(E)=\sum_{(m \alpha)}\left[g(m \alpha ; E) C^{+}(m \alpha)-h(m \alpha ; E) C(m \alpha)\right] \text {. }
$$

Examination of the commutators in Eqs. (3a) and (17) shows that this approximation takes into account all of the single particle-hole terms. In this case, also, there will be an equation analogous to Eq. (14) involving $C(m \alpha)$.

Using the resulting Eqs. (14) and the commutators for the particle-hole operators, we get the set of coupled equations

$$
\begin{aligned}
& {\left[\epsilon_{m}-\epsilon_{\alpha}+\mathrm{V}_{m \alpha \alpha m}-\mathrm{V}_{m \alpha m \alpha}-\left(E-E_{0}\right)\right] g(m \alpha)+\sum_{(n \beta)}^{\prime}\left(\mathrm{V}_{\alpha n m \beta}-\mathrm{V}_{\alpha n \beta m}\right) g(n \beta)+\sum_{(n \beta)}\left(\mathrm{V}_{\alpha \beta m n}-\mathrm{V}_{\alpha \beta n m}\right) h(n \beta)=0,} \\
& {\left[\epsilon_{m}-\epsilon_{\alpha}+\mathrm{V}_{m \alpha \alpha m}-\mathrm{V}_{m \alpha m \alpha}+\left(E-E_{0}\right)\right] h(m \alpha)+\sum_{(n \beta)}^{\prime}\left(\mathrm{V}_{\alpha n m \beta}-\mathrm{V}_{\alpha n \beta m}\right) h(n \beta)+\sum_{(n \beta)}\left(\mathbf{V}_{\alpha \beta m n}-\mathbf{V}_{\alpha \beta n m}\right) g(n \beta)=0}
\end{aligned}
$$

Specializing these equations for singlets and triplets in the usual way leads to for singlets:

$$
\begin{aligned}
& {\left[\epsilon_{m}-\epsilon_{\alpha}+2 V_{m \alpha \alpha m}-V_{m \alpha m \alpha}-\left({ }^{1} E-E_{0}\right)\right] g(m \alpha)+\sum_{(n \beta)}^{\prime}\left(2 V_{\alpha n m \beta}-V_{\alpha n \beta m}\right) g(n \beta)+\sum_{(n \beta)}\left(2 V_{\alpha \beta m n}-V_{\alpha \beta n m}\right) h(n \beta)=0,} \\
& {\left[\epsilon_{m}-\epsilon_{\alpha}+2 V_{m \alpha \alpha m}-V_{m \alpha m \alpha}+\left({ }^{1} E-E_{0}\right)\right] h(m \alpha)+\sum_{(n \beta)}^{\prime}\left(2 V_{\alpha n m \beta}-V_{\alpha n \beta m}\right) h(n \beta)+\sum_{(n \beta)}\left(2 V_{\alpha \beta m n}-V_{\alpha \beta n m}\right) g(n \beta)=0,}
\end{aligned}
$$

and for triplets:

$$
\begin{aligned}
& {\left[\epsilon_{m}-\epsilon_{\alpha}-V_{m \alpha m \alpha}-\left({ }^{3} E-E_{0}\right)\right] g(m \alpha)-\sum_{(n \beta)}^{\prime} V_{\alpha n \beta m} g(n \beta)-\sum_{(n \beta)} V_{\alpha \beta n m} h(n \beta)=0,} \\
& {\left[\epsilon_{m}-\epsilon_{\alpha}-V_{m \alpha m \alpha}+\left({ }^{3} E-E_{0}\right)\right] h(m \alpha)-\sum_{(n \beta)}^{\prime} V_{\alpha n \beta m} h(n \beta)-\sum_{(n \beta)} V_{\alpha \beta n m} g(n \beta)=0 .}
\end{aligned}
$$

These equations can be written in matrix notation as

$$
\left(\begin{array}{cc}
\mathbf{A} & \mathbf{B} \\
-\mathbf{B} & -\mathbf{A}
\end{array}\right)\left(\begin{array}{l}
\mathbf{G} \\
\mathbf{H}
\end{array}\right)=\Delta E\left(\begin{array}{l}
\mathbf{G} \\
\mathrm{H}
\end{array}\right),
$$

where for the singlet state

$$
\begin{aligned}
& \mathbf{A}(m \alpha, m \alpha)=\epsilon_{m}-\epsilon_{\alpha}+2 V_{m \alpha \alpha m}-V_{m \alpha m \alpha}, \\
& \mathbf{A}(m \alpha, n \beta)=2 V_{\alpha n m \beta}-V_{\alpha n \beta m}, \\
& \mathbf{B}(m \alpha, n \beta)=2 V_{\alpha \beta m n}-V_{\alpha \beta n m},
\end{aligned}
$$

and a corresponding set for the triplet.

Comparing the above equations to those derived in the previous section, we note that the TDA involves only the matrix $A$, and the STA only the diagonal elements of $A$. The B matrix allows for the effect of the doubly excited components of the true ground state on the singly excited state.

From Eq. (32) we see that the excitation energies are the eigenvalues of a non-Hermitian matrix. Because of this the eigenvalues and eigenvectors have some peculiar properties which we now discuss.

The matrix form of the RPA equations indicates that negative eigenvalues occur with the same magnitude as the positive eigenvalues. To see this, take the Hermitian conjugate of Eq. (32) to get

$$
\left(\begin{array}{rr}
\mathbf{A} & \mathbf{B} \\
-\mathbf{B} & -\mathbf{A}
\end{array}\right)\left(\begin{array}{c}
\mathbf{H} \\
\mathbf{G}
\end{array}\right)=-\Delta E\left(\begin{array}{l}
\mathbf{H} \\
\mathbf{G}
\end{array}\right) \text {. }
$$

Thus, the eigenvectors for the negative eigenvalues are identical to those of the positive eigenvalues with the roles of $\mathbf{H}$ and $\mathbf{G}$ interchanged. The requirement that the excited states be orthonormal

leads to

$$
\left\langle E \mid E^{\prime}\right\rangle=\delta_{E E^{\prime}}
$$

or

$$
\left\langle 0\left|\left[A(E), A^{+}\left(E^{\prime}\right)\right]\right| 0\right\rangle=\delta_{E E^{\prime}}
$$

$$
\sum\left[g(m \alpha ; E) g\left(m \alpha ; E^{\prime}\right)-h(m \alpha ; E) h\left(m \alpha ; E^{\prime}\right)\right]=\delta_{E E^{\prime}} .
$$

The particle-hole amplitudes are, thus, normalized to an indefinite metric. Last, we note that the excitation energies could be complex. However, because of the physical interpretation of these eigenvalues we are only concerned with those which have $\operatorname{Im}(\Delta E)=0$.

The above is the random-phase approximation as it usually is presented in the literature. ${ }^{5,6,8} \mathrm{We}$ would now like to pause and elaborate on the derivation of the RPA equations. The origin of the non-Hermiticity of the matrix then becomes apparent.

In deriving the RPA equations we have assumed for the ground state to first order

that

$$
|0\rangle=C_{0}|\mathrm{HF}\rangle+\sum C_{d}|d\rangle
$$

$$
C_{0} \approx 1, \quad C_{d} \ll 1 .
$$

This allows us to use the ground-state approximation, 
TABLE II. Wavefunction of ethylene."

\begin{tabular}{|c|c|c|c|c|c|c|c|c|}
\hline \multirow[b]{2}{*}{$\mathrm{MO}^{\mathrm{b}}$} & \multirow[b]{2}{*}{$\begin{array}{l}\text { Orbital } \\
\text { energy }\end{array}$} & \multicolumn{7}{|c|}{ Coefficients } \\
\hline & & $\underset{2 p_{x} \mathrm{C} 1}{\mathrm{H} 1}$ & $\underset{2 p y \mathrm{C} 1}{\mathrm{H} 2}$ & $\begin{array}{c}\mathrm{H3} \\
1 s \mathrm{C} 2\end{array}$ & $\begin{array}{c}\mathrm{H} 4 \\
2 s \mathrm{C} 2\end{array}$ & $\begin{array}{c}1 s \mathrm{C} 1 \\
2 p_{2} \mathrm{C} 2\end{array}$ & $\begin{array}{l}2 s \mathrm{C} 1 \\
2 p_{x} \mathrm{C} 2\end{array}$ & $\begin{array}{l}2 p_{s} \mathrm{C} 1 \\
2 p_{y} \mathrm{C} 2\end{array}$ \\
\hline $1 a_{g}\left(1 a_{g}\right)$ & -11.3391 & $\begin{array}{l}-0.004420 \\
0 .\end{array}$ & $\begin{array}{l}-0.004420 \\
0 .\end{array}$ & $\begin{array}{r}-0.004420 \\
0.703982\end{array}$ & $\begin{array}{r}-0.004420 \\
0.014810\end{array}$ & $\begin{array}{l}0.703982 \\
0.002067\end{array}$ & $\begin{array}{l}0.014810 \\
0 .\end{array}$ & $\begin{array}{l}-0.002067 \\
0 .\end{array}$ \\
\hline $1 b_{1 u}\left(1 b_{3 u}\right)$ & -11.3384 & $\begin{array}{l}-0.004461 \\
0 .\end{array}$ & $\begin{array}{l}-0.004461 \\
0 .\end{array}$ & $\begin{array}{r}0.004461 \\
-0.703805\end{array}$ & $\begin{array}{r}0.004461 \\
-0.023659\end{array}$ & $\begin{array}{l}0.703805 \\
0.003673\end{array}$ & $\begin{array}{l}0.023659 \\
0 .\end{array}$ & $\begin{array}{l}0.003673 \\
0 .\end{array}$ \\
\hline $2 a_{0}\left(2 a_{g}\right)$ & -1.0419 & $\begin{array}{l}0.080301 \\
0 .\end{array}$ & $\begin{array}{l}0.080301 \\
0 .\end{array}$ & $\begin{array}{r}0.080301 \\
-0.161837\end{array}$ & $\begin{array}{l}0.080301 \\
0.479569\end{array}$ & $\begin{array}{l}-0.161837 \\
-0.110549\end{array}$ & $\begin{array}{l}0.479569 \\
0 .\end{array}$ & $\begin{array}{l}0.110549 \\
0 .\end{array}$ \\
\hline $2 b_{1 u}\left(2 b_{3 u}\right)$ & -0.8025 & $\begin{array}{l}0.206081 \\
0 .\end{array}$ & $\begin{array}{l}0.206081 \\
0 .\end{array}$ & $\begin{array}{r}-0.206081 \\
0.126147\end{array}$ & $\begin{array}{l}-0.206081 \\
-0.431860\end{array}$ & $\begin{array}{l}-0.126147 \\
-0.203934\end{array}$ & $\begin{array}{l}0.431860 \\
0 .\end{array}$ & $\begin{array}{l}-0.203934 \\
0 .\end{array}$ \\
\hline $1 b_{3 u}\left(1 b_{2 u}\right)$ & -0.6661 & $\begin{array}{l}0.251413 \\
0.399054\end{array}$ & $\begin{array}{l}-0.251413 \\
0 .\end{array}$ & $\begin{array}{l}0.251413 \\
0 .\end{array}$ & $\begin{array}{l}-0.251413 \\
0 .\end{array}$ & $\begin{array}{l}0 . \\
0 .\end{array}$ & $\begin{array}{l}0 . \\
0.399054\end{array}$ & $\begin{array}{l}\mathbf{0} . \\
\mathbf{0}\end{array}$ \\
\hline $3 a_{g}\left(3 a_{q}\right)$ & -0.5908 & $\begin{array}{l}-0.207729 \\
0 .\end{array}$ & $\begin{array}{l}-0.207729 \\
0 .\end{array}$ & $\begin{array}{l}-0.207729 \\
-0.011250\end{array}$ & $\begin{array}{r}-0.207729 \\
0.025045\end{array}$ & $\begin{array}{l}-0.011250 \\
-0.512543\end{array}$ & $\begin{array}{l}0.025045 \\
0 .\end{array}$ & $\begin{array}{l}0.512543 \\
0 .\end{array}$ \\
\hline $1 b_{20}\left(1 b_{10}\right)$ & -0.5292 & $\begin{array}{l}0.367882 \\
0.408586\end{array}$ & $\begin{array}{l}-0.367882 \\
0 .\end{array}$ & $\begin{array}{l}-0.367882 \\
0 .\end{array}$ & $\begin{array}{l}0.367882 \\
0 .\end{array}$ & $\begin{array}{l}0 . \\
0 .\end{array}$ & $\begin{array}{l}0 . \\
-0.408586\end{array}$ & $\begin{array}{l}\mathbf{0} . \\
0 .\end{array}$ \\
\hline $1 b_{2 w}\left(1 b_{1 w}\right)$ & -0.4047 & 0. & $\begin{array}{l}0 . \\
0.628564\end{array}$ & $\begin{array}{l}0 . \\
0 .\end{array}$ & $\begin{array}{l}0 . \\
0 .\end{array}$ & $\begin{array}{l}0 . \\
0 .\end{array}$ & $\begin{array}{l}0 . \\
0 .\end{array}$ & 0.628564 \\
\hline $1 b_{3 q}\left(1 b_{2 q}\right)$ & 0.2056 & $\begin{array}{l}0 . \\
0 .\end{array}$ & $\begin{array}{l}0 . \\
0.825081\end{array}$ & $\begin{array}{l}0 . \\
0 .\end{array}$ & $\begin{array}{l}0 . \\
0 .\end{array}$ & $\begin{array}{l}0 . \\
0 .\end{array}$ & $\begin{array}{l}0 . \\
0 .\end{array}$ & $\begin{array}{l}0 . \\
-0.825081\end{array}$ \\
\hline $4 a_{g}\left(4 a_{\theta}\right)$ & 0.4344 & $\begin{array}{l}-0.669375 \\
0 .\end{array}$ & $\begin{array}{l}-0.669375 \\
0 .\end{array}$ & $\begin{array}{l}-0.669375 \\
-0.101083\end{array}$ & $\begin{array}{r}-0.669375 \\
0.955990\end{array}$ & $\begin{array}{r}-0.101083 \\
0.515356\end{array}$ & $\begin{array}{l}0.995990 \\
0 .\end{array}$ & $\begin{array}{l}-0.515356 \\
0 .\end{array}$ \\
\hline $2 b_{3 u}\left(2 b_{2 u}\right)$ & 0.4409 & $\begin{array}{r}0.711080 \\
-0.756979\end{array}$ & $\begin{array}{l}-0.711080 \\
0 .\end{array}$ & $\begin{array}{l}0.711080 \\
0 .\end{array}$ & $\begin{array}{l}-0.711080 \\
0 .\end{array}$ & $\begin{array}{l}0 . \\
0 .\end{array}$ & $\begin{array}{l}0 . \\
-0.756979\end{array}$ & $\begin{array}{l}0 . \\
0 .\end{array}$ \\
\hline $3 b_{14}\left(3 b_{3 u}\right)$ & 0.4711 & $\begin{array}{l}0.725739 \\
0 .\end{array}$ & $\begin{array}{l}0.725739 \\
0 .\end{array}$ & $\begin{array}{l}-0.725739 \\
-0.132831\end{array}$ & $\begin{array}{r}-0.725739 \\
1.153837\end{array}$ & $\begin{array}{l}0.132831 \\
0.270117\end{array}$ & $\begin{array}{l}-1.153837 \\
0 .\end{array}$ & $\begin{array}{l}0.270117 \\
0 .\end{array}$ \\
\hline $2 b_{2 \sigma}\left(2 b_{10}\right)$ & 0.7179 & $\begin{array}{r}0.727053 \\
-1.019986\end{array}$ & $\begin{array}{l}-0.727053 \\
0 .\end{array}$ & $\begin{array}{l}-0.727053 \\
0 .\end{array}$ & $\begin{array}{l}0.727053 \\
0 .\end{array}$ & $\begin{array}{l}0 . \\
0\end{array}$ & 0.019986 & $\begin{array}{l}0 . \\
0 .\end{array}$ \\
\hline $4 b_{1 u}\left(4 b_{3 w}\right)$ & 0.8037 & $\begin{array}{l}0.105834 \\
0 .\end{array}$ & $\begin{array}{l}0.105834 \\
0 .\end{array}$ & $\begin{array}{r}-0.105834 \\
0.094518\end{array}$ & $\begin{array}{l}-0.105834 \\
-0.992343\end{array}$ & $\begin{array}{r}-0.094518 \\
1.210833\end{array}$ & $\begin{array}{l}0.992343 \\
0 .\end{array}$ & $\begin{array}{l}1.210833 \\
0 .\end{array}$ \\
\hline
\end{tabular}

a This table is from unpublished calculations by W. E. Palke and W. N, Lipscomb. To facilitate comparison with their previously published results, Ref. 3 , the molecule here lies in the $x z$ plane. For the remainder of this work the molecule has been rotated into the $x y$ plane to agree with the usual spectroscopic notation.

i.e., ignore products of the $C_{d}$ 's while retaining the terms linear in $C_{d}$. To evaluate the commutators in Eq. (14) over the state $|0\rangle$, we can then effectively use the Hartree-Fock ground state. Thus, only if the HF ground state closely represents the true ground state can we expect the RPA results to be valid.

From the form of the singlet and triplet RPA equations, we can see that the RPA is more likely to fail in locating a triplet than a singlet excited state. Specifically, for molecular calculations the appearance of this "instability" will depend on how closely the LCAO expansion approaches the HF limit; although in some pathological cases of strong mixing of the ground state with a doubly excited configuration, it may never disappear. Fortunately, the correction of this defect is straightforward: one need merely evaluate the commutators using the ground-state wavefunction to first order. This, however, assumes that the correlation coefficients, $C_{d}$, are known-just the difficulty that we b The symmetry of the orbitals for the molecule rotated into the $x y$ plane are enclosed in parentheses. Note that in Ref. 3 , the $b_{1 u}$ orbitals were incorrectly labeled as $a\llcorner u$ (private communication from W. E. Palke).

were trying to bypass when we used the ground-state approximation. There is another, and more interesting, approach to the problem. From the definition of the excitation operator, we find that there is a mutual constraint which this operator and the ground-state wavefunction must satisfy, namely,

$$
A(E)|0\rangle=0 .
$$

Given the form of the excitation operator, we merely look for a ground state which satisfies the above relation. For the RPA excitation operator the ground state is as given in Eq. (28) and equations can be derived which permit the calculation of the correlation coefficients, $C_{\alpha \beta}{ }^{m n} .^{22}$ In nuclear physics this is known as the extended RPA. ${ }^{23}$ Its applicability to molecular systems is presently under study.

\footnotetext{
22 E. A. Sanderson, Phys. Letters 19, 141 (1965).

${ }_{23}$ K. Hara, Progr. Theoret. Phys. Kyoto 32, 88 (1964); K. Ikeda, T. Udagawa, and H. Yamaura, ibid. 33, 22 (1965).
} 
NONEMPIRICAL CALCULATIONS ON EXCITED STATES

TaBLE III. The molecular integrals, $V_{i j k l}$, required for the treatment of the $B_{3 u}$ state of ethylene. ${ }^{\text {s }}$

\begin{tabular}{|c|c|c|c|c|c|c|c|c|c|}
\hline$i$ & $j$ & $k$ & $l$ & $V_{i j k l}$ & $i$ & $j$ & $k$ & $l$ & $V_{i j k l}$ \\
\hline 9 & 9 & 8 & 8 & 0.486677 & 12 & 6 & 12 & 6 & 0.025382 \\
\hline 11 & 9 & 8 & 7 & -0.006293 & 14 & 6 & 12 & 6 & 0.010080 \\
\hline 12 & 9 & 8 & 6 & -0.000596 & 12 & 6 & 10 & 4 & 0.029903 \\
\hline 14 & 9 & 8 & 6 & 0.018200 & 13 & 5 & 12 & 6 & -0.016009 \\
\hline 10 & 9 & 8 & 4 & 0.024901 & 12 & 6 & 12 & 3 & -0.014651 \\
\hline 13 & 9 & 8 & 5 & -0.011451 & 14 & 3 & 12 & 6 & -0.009848 \\
\hline 12 & 9 & 8 & 3 & -0.046558 & 14 & 6 & 14 & 6 & 0.089809 \\
\hline 14 & 9 & 8 & 3 & 0.043314 & 14 & 6 & 10 & 4 & 0.045175 \\
\hline 11 & 11 & 7 & 7 & 0.358632 & 14 & 6 & 13 & 5 & -0.039177 \\
\hline 12 & 11 & 7 & 6 & -0.034885 & 14 & 6 & 12 & 3 & -0.039565 \\
\hline 14 & 11 & 7 & 6 & -0.002028 & 14 & 6 & 14 & 3 & 0.047390 \\
\hline 11 & 10 & 7 & 4 & -0.055069 & 10 & 4 & 10 & 4 & 0.050754 \\
\hline 13 & 11 & 7 & 5 & 0.117438 & 13 & 5 & 10 & 4 & -0.034288 \\
\hline 12 & 11 & 7 & 3 & 0.037165 & 12 & 3 & 10 & 4 & -0.033584 \\
\hline 14 & 11 & 7 & 3 & -0.014081 & 14 & 3 & 10 & 4 & 0.009595 \\
\hline 12 & 12 & 6 & 6 & 0.360222 & 13 & 5 & 13 & 5 & 0.048769 \\
\hline 14 & 12 & 6 & 6 & -0.015710 & 11 & 7 & 11 & 7 & 0.044318 \\
\hline 12 & 10 & 6 & 4 & 0.109642 & 12 & 6 & 11 & 7 & -0.020512 \\
\hline 13 & 12 & 6 & 5 & -0.027352 & 11 & 8 & 9 & 7 & -0.007751 \\
\hline 12 & 12 & 6 & 3 & 0.009487 & 12 & 8 & 9 & 6 & 0.006714 \\
\hline 14 & 12 & 6 & 3 & -0.035274 & 14 & 8 & 9 & 6 & 0.008209 \\
\hline 14 & 13 & 6 & 5 & -0.007745 & 10 & 8 & 9 & 4 & 0.027503 \\
\hline 14 & 12 & 6 & 3 & -0.035274 & 13 & 8 & 9 & 5 & -0.009673 \\
\hline 14 & 14 & 6 & 3 & 0.111471 & 12 & 8 & 9 & 3 & -0.030172 \\
\hline 10 & 10 & 4 & 4 & 0.359020 & 14 & 8 & 9 & 3 & 0.024716 \\
\hline 13 & 10 & 5 & 4 & -0.039745 & 12 & 7 & 11 & 6 & -0.023760 \\
\hline 12 & 10 & 4 & $\overline{3}$ & -0.094796 & 14 & 7 & 11 & 6 & -0.017414 \\
\hline 14 & 10 & 4 & 3 & -0.036816 & 11 & 4 & 10 & 7 & -0.022275 \\
\hline 13 & 13 & 5 & 5 & 0.391534 & 13 & 7 & 11 & 5 & 0.061154 \\
\hline 13 & 12 & 5 & 3 & 0.055546 & 12 & 7 & 11 & 3 & 0.015003 \\
\hline 14 & 13 & 5 & 3 & -0.033708 & 14 & 7 & 11 & 3 & -0.018506 \\
\hline 12 & 12 & 3 & 3 & 0.359503 & 12 & 4 & 10 & 6 & 0.042439 \\
\hline 14 & 12 & 3 & 3 & -0.040165 & 13 & 6 & 12 & 5 & -0.013004 \\
\hline 14 & 14 & 3 & 3 & 0.537546 & 12 & 6 & 12 & 3 & -0.014651 \\
\hline 9 & 8 & 9 & 8 & 0.158394 & 14 & 6 & 12 & 3 & -0.039565 \\
\hline 11 & 7 & 9 & 8 & -0.014897 & 14 & 4 & 10 & 6 & 0.016506 \\
\hline 12 & 6 & 9 & 8 & 0.009200 & 14 & 5 & 13 & 6 & -0.009401 \\
\hline 14 & 6 & 9 & 8 & 0.092648 & 14 & 3 & 12 & 6 & -0.009848 \\
\hline 10 & 4 & 9 & 8 & 0.056091 & 14 & 6 & 14 & 3 & 0.047390 \\
\hline 13 & 5 & 9 & 8 & -0.050457 & 13 & 5 & 12 & 3 & 0.032140 \\
\hline 12 & 3 & 9 & 8 & -0.055192 & 14 & 3 & 13 & 5 & -0.034339 \\
\hline 14 & 3 & 9 & 8 & 0.060550 & 12 & 3 & 12 & 3 & 0.029072 \\
\hline 14 & 14 & 6 & 6 & 0.499948 & 14 & 3 & 12 & 3 & -0.025136 \\
\hline 14 & 10 & 6 & 4 & 0.048800 & 14 & 3 & 14 & 3 & 0.060354 \\
\hline 14 & 6 & 11 & 7 & -0.014189 & 13 & 4 & 10 & 5 & -0.016829 \\
\hline 11 & 7 & 10 & 4 & -0.027126 & 12 & 4 & 10 & 3 & -0.030548 \\
\hline 13 & 5 & 11 & 7 & 0.039152 & 14 & 4 & 10 & 3 & -0.009334 \\
\hline 12 & 3 & 11 & 7 & 0.023518 & 13 & 3 & 12 & 5 & 0.014030 \\
\hline 14 & 3 & 11 & 7 & -0.019405 & 14 & 5 & 13 & 3 & -0.015973 \\
\hline
\end{tabular}

${ }^{\text {a }}$ In this table

$V_{i j k l}=\iint \phi_{i}^{*}(1) \phi_{j}(1) r_{12}{ }^{-1} \phi_{k}^{*}(2) \phi_{1}(2) d \eta d v_{2}$.

This arrangement was chosen to coincide with that of the atomic integrals obtained from the LCAO MO SF calculation. The integrals are in atomic units.

For the RPA, the transition moment is

$$
{ }^{1} \mathrm{D}_{\mathrm{RPA}}=-\sqrt{2} \sum_{(m \alpha)}[g(m \alpha)+h(m \alpha)] \mathbf{d}_{m \alpha} .
$$

For comparison with experiment we also calculate the oscillator strength of a transition. The oscillator strength, $f$, is defined by

$$
f=\frac{2}{3} \Delta E|\mathrm{D}|^{2},
$$

where $\Delta E$ is the theoretically calculated excitation energy, and $\Delta E$ and $\mathrm{D}$ are both in atomic units.
Before closing this section we should point out that the RPA equations can be derived via time-dependent Hartree-Fock theory (TDHF) ${ }^{24,26}$ However, the interpretation of the resulting wavefunctions from that viewpoint is not at all clear. ${ }^{26}$ Also, the remedy of a breakdown in the theory, corresponding to the instability discussed here, cannot be implemented so easily as in the present approach.

${ }^{24}$ M. A. Ball and A. D. McLachlan, Mol. Phys. 7, 501 (1964). ${ }^{25}$ A. D. McLachlan, Rev. Mod. Phys. 36, 844 (1964).

${ }^{26}$ D. J. Rowe, Nucl. Phys. 80, 209 (1966). 
TaBLE IV. The single-particle transitions coupled to form the excited $B_{3 u}$ states of ethylene.

\begin{tabular}{lclc}
\hline \multicolumn{2}{c}{ Transition } & & \multicolumn{1}{c}{ Approximate } \\
Symmetry & Numerical & & $\begin{array}{c}\text { Singlet } \\
\text { description }\end{array}$ \\
\hline $1 b_{1 u} \rightarrow 1 b_{2 g}$ & $8 \rightarrow 9$ & $\pi \rightarrow \pi^{*}$ & $\begin{array}{c}\text { transition } \\
\text { energy }\end{array}$ \\
$1 b_{1 g} \rightarrow 2 b_{2 u}$ & $7 \rightarrow 11$ & $\mathrm{CH} \rightarrow \mathrm{CH}^{*}$ & 11.98 \\
$3 a_{g} \rightarrow 3 b_{3 u}$ & $6 \rightarrow 12$ & $\mathrm{CH}(\sigma) \rightarrow \mathrm{CH}^{*}\left(\sigma^{*}\right)$ & 19.05 \\
$3 a_{g} \rightarrow 4 b_{3 u}$ & $6 \rightarrow 14$ & $\mathrm{CH}(\sigma) \rightarrow \sigma^{*}$ & 20.47 \\
$2 b_{3 u} \rightarrow 4 a_{g}$ & $4 \rightarrow 10$ & $\mathrm{CH}\left(\sigma^{*}\right) \rightarrow \mathrm{CH}^{*}(\sigma)$ & 29.23 \\
$1 b_{2 u} \rightarrow 2 b_{1 g}$ & $5 \rightarrow 13$ & $\mathrm{CH} \rightarrow \mathrm{CH}^{*}$ & 29.65 \\
$2 a_{g} \rightarrow 3 b_{3 u}$ & $3 \rightarrow 12$ & $\sigma \rightarrow \mathrm{CH}^{*}\left(\sigma^{*}\right)$ & 32.97 \\
$2 a_{\sigma} \rightarrow 4 b_{3 u}$ & $3 \rightarrow 14$ & $\sigma \rightarrow \sigma^{*}$ & 38.88 \\
\hline
\end{tabular}

The symmetry designation is that with the molecule in the $x y$ plane. The numerical designation is used in the following table to identify the two-electron integrals.

b Except for the first transition, these descriptions are only qualitative since the orbitals are delocalized. The designation enclosed in parentheses refers to the minor component of the orbital.

- Calculated with Eq. (21a) of the text. The excitation energies are in electron volts.

\section{RESULTS}

To provide a concrete foundation for the theories discussed in this paper, we have carried out the full set of calculations on the ethylene molecule using the recent LCAO MO SCF wavefunction of Palke and Lipscomb (unpublished results, see Table II). The purposes of the calculation are: (1) to check the relevance of these theories for the calculation of the electronic spectra of molecular systems and (2) to rigorously investigate the proposal of Herzenberg et al. ${ }^{9}$ that the coupling between the $\sigma$ and $\pi$ electrons in ethylene could have a profound effect on the lowestlying excited states of that molecule. Their results were obtained in the TDA with a dipole-dipole approximation for $1 / r_{12}$ and a semiempirical evaluation of the integrals which caused some skepticism about the strength of the observed coupling.

In Table II the wavefunction obtained by Palke and Lipscomb ${ }^{12}$ for ethylene is given. The basis functions are Slater orbitals with orbitals exponents chosen by Slater's rules; this is in contrast to their published work ${ }^{3}$ in which a hydrogen exponent of 1.2 was used. Table III lists the two-electron molecular integrals, $V_{i j k l}$, required for the calculation on the $B_{3 u}$ state. The atomic integrals are available upon request.

In Table IV are listed the eight excitations which are coupled together to form the excited $B_{3 u}$ states of ethylene. We have ignored any excitation of the $1 \mathrm{~s}$ electrons as being energetically unfavorable; calculations on the other states showed that the contribution from the $1 s$ electrons was indeed negligible.

Tables V and VI give the results of the calculations on the singlet and triplet states of symmetry $B_{3 u}$. Excitation energies, transition moments, oscillator strengths, and wavefunctions (particle-hole amplitudes) for the STA, TDA, and RPA are given.
The STA values are comparable to those presented earlier for the $\pi$-electron calculations for the same assumptions are inherent in both, namely, a neglect of the effect of the excitation of a $\pi$ electron on the remaining electrons in the molecule (the $\sigma$ electrons in this case). The only difference is that in the LCAO MO SCF calculation the core potential is generated exactly (within the limited basis set used) in the ground-state calculation.

From the TDA results we immediately note the profound effect that the inclusion of the $\sigma$ electrons has on both the excitation energy and the oscillator strength. It is just not possible to ignore the rearrangement of the $\sigma$ core as is done in $\pi$-electron theory. It is quite polarizable. From the wavefunctions we can see why this interaction was not detected earlier. In the previous calculations which attempted to answer the question of $\sigma-\pi$ separability, ${ }^{27}$ the $\sigma$ electrons which were considered were those of the carbon-carbon bond. Clearly, the electrons to be considered are those in the $\mathrm{CH}$ bonds for these are the ones most strongly coupled to the $\pi \rightarrow \pi^{*}$ transition.

As expected, the RPA results are merely a refinement of those of the TDA, bringing the calculated excitation

TABLE V. The lowest singlet state of ethylene of symmetry $B_{3 u}$ : excitation energies (in electron volts), transition moments, oscillator strengths, and wavefunctions for the various approximations to the excited state.

\begin{tabular}{crrl}
\hline & STA $^{\mathrm{a}}$ & TDA $^{\mathrm{b}}$ & RPAa \\
\hline$\Delta E$ & 11.98 & 10.17 & 9.44 \\
$D^{\mathrm{d}}$ & 1.87 & 1.71 & 1.49 \\
$f$ & 1.03 & 0.73 & 0.51
\end{tabular}

Wavefunctions

$\begin{array}{clrr}(m, \alpha) & g(m \alpha) & g(m \alpha) & g(m \alpha) \\ (9,8) & 1.000000 & 0.960329 & 0.966983 \\ (11,7) & & 0.056820 & 0.036436 \\ (12,6) & & -0.033354 & -0.023823 \\ (14,6) & & -0.206246 & -0.160605 \\ (10,4) & & -0.119678 & -0.087569 \\ (13,5) & & 0.093880 & 0.067878 \\ (12,3) & & 0.062356 & 0.041913 \\ (14,3) & & -0.062177 & -0.045988 \\ & & & h(m \alpha) \\ (9,8) & & & -0.098574 \\ (11,7) & & & 0.006566 \\ (12,6) & & & 0.000955 \\ (14,6) & & & -0.083727 \\ (10,4) & & & -0.032685 \\ (13,5) & & & 0.032538 \\ (12,3) & & & 0.029633 \\ (14,3) & & & -0.036484\end{array}$

From Eqs. (21a), (22a), and (38) of the text.

From Eqs. (25a), (26), and (38) of the text.

- From Eqs. (31a), (31b), (37), and (38) of the text.

d In atomic units. The dipole matrix elements were computed using the dipole-moment program of M. D. Newton and F. P. Boer as modified by R. Frank.

Unnormalized, $\Sigma\left[g^{2}(m \alpha)-h^{2}(m \alpha)\right]=0.957785$; see Eq. (35c) of the text.

${ }^{27}$ C. M. Moser, Trans. Faraday Soc. 49, 1239 (1953). 
energy and oscillator strength into better agreement with experiment.

Because of the large

$$
\left(\begin{array}{cc}
\pi^{*} & \bar{\pi}^{*} \\
& \bar{\pi}
\end{array}\right)
$$

component in the ground state, see Table VII, the conditions for the application of the RPA were not strictly satisfied and, as a result, the excitation energy of the triplet state became pure imaginary. The remedy of this situation, if it is worthwhile since the properties of triplet states are relatively easy to predict with just the STA, has been discussed previously and is not commented on further here. It is instructive to note, however, that the wavefunction of Moskowitz and Harrison, ${ }^{17}$ which they believe approaches the $(s p)$ limit, does not exhibit this instability.

TABLE VI. The lowest triplet state of ethylene of symmetry $B_{3 u}$ : excitation energies (in electron volts) and wavefunctions for the various approximations to the excited state.

\begin{tabular}{clr}
\hline & \multicolumn{1}{c}{ STA $^{\mathbf{a}}$} & \multicolumn{1}{c}{ TDA $^{\mathrm{b}}$} \\
\hline$\Delta E$ & 3.36 & 3.19 \\
Wavefunctions & & \\
$(m, \alpha)$ & $g(m \alpha)$ & $g(m \alpha)$ \\
$(9,8)$ & 1.000000 & 0.995423 \\
$(11,7)$ & & -0.029092 \\
$(12,6)$ & & 0.005806 \\
$(14,6)$ & & 0.034536 \\
$(10,4)$ & & 0.043743 \\
$(13,5)$ & & -0.024380 \\
$(12,3)$ & & -0.053773 \\
$(14,3)$ & & 0.040760 \\
\hline
\end{tabular}

From Eq. (21b) of the text.

b From Eq. (25b) of the text.

In these calculations we did not make the assumptions and approximations that Herzenberg et al. ${ }^{9}$ did. But, our results indicate that their conclusions are essentially correct. As a further test of their suggestion ${ }^{9}$ that the effect of the particle-hole scattering terms is negligible, the calculation for the $B_{3 u}$ state was done neglecting those terms. The results were essentially the same as those obtained previously: the contribution from the $\sigma$ core being appreciable.

The effect obtained for the transition moments is particularly striking. It has long been known that transition moments calculated by the simple MO method (STA) are off by a factor of 2 or so-the explanation being electronic correlation. As evidenced by this calculation, the inclusion of all particle-hole terms provides an adequate explanation of the above phenomena. The discrepancy is a result of neglecting the "unexcited" electrons in the molecule, e.g., the $\sigma$ electrons in ethylene during a $\pi$-electron transition.
TABLE VII. Configuration interaction: inclusion of the

$$
\left(\begin{array}{ll}
\pi^{*} & \bar{\pi}^{*} \\
\pi & \bar{\pi}
\end{array}\right)
$$

component in the ground state of ethylene.

$$
\begin{aligned}
& \text { Required integrals (in a.u.) } \\
& \epsilon_{\pi}=-0.4047 \quad \epsilon_{\pi}^{*}=0.2056 \\
& J_{\pi x}=0.487271 \\
& J_{\pi}{ }^{*}{ }^{*}=0.500717 \\
& J_{\pi \pi}^{*}=0.486677 \quad K_{\pi \pi^{*}}=0.158394 \\
& \text { Results } \\
& E-E_{\mathrm{HF}}=-0.040518 \text { a.u. } \\
& \text { Coefficients } \\
& \begin{array}{ll}
C_{0} & 0.968805
\end{array} \\
& C\left(\begin{array}{ll}
\pi^{*} & \bar{\pi}^{*} \\
\pi & \bar{\pi}
\end{array}\right) \quad-0.247825
\end{aligned}
$$

The program for diagonalizing the unsymmetric RPA matrix was provided by S. F. Persselin of Rocketdyne Division of the North American Aviation Company. With this program we were able to reproduce the required symmetry of the eigenvectors to eight significant figures by suitable adjustment of the optimization parameters and, hence, we feel that the performance of the program is satisfactory.

Finally, in Table VIII the lowest excitation energies for states of various symmetries are listed. Of particular interest are the two low-lying states of symmetry $B_{\mathbf{2}}$ and $B_{30}$ which are also in the same region of the spectrum as the singlet $B_{3 u}$ state.

\section{DISCUSSION AND CONCLUSIONS}

These results indicate that most of the essential features of an excitation are contained in the TDA with the RPA merely altering these values by small, but far from negligible, amounts. Within the scope of the TDA

TABLE VIII. Excitation energies for other low-lying excited states of ethylene.

\begin{tabular}{crrr}
\hline & \multicolumn{2}{c}{$\begin{array}{c}\text { Excitation energies } \\
\text { (in eV) }\end{array}$} \\
\cline { 2 - 4 } State & \multicolumn{1}{c}{ STA $^{\mathbf{a}}$} & TDA $^{\mathbf{b}}$ & \multicolumn{1}{c}{ RPA $^{\mathrm{c}}$} \\
\hline${ }^{3} B_{2 g}$ & 9.99 & 9.73 & 9.43 \\
${ }^{1} B_{2 g}$ & 10.76 & 10.47 & 10.32 \\
${ }^{3} B_{3 g}$ & 9.82 & 9.81 & 9.79 \\
${ }^{1} B_{3_{g}}$ & 10.46 & 10.44 & 10.44 \\
${ }^{3} B_{1 u}$ & 13.23 & 13.10 & 12.96 \\
${ }^{1} B_{1 u}$ & 14.24 & 14.11 & 14.10 \\
${ }^{3} B_{2 u}$ & 17.78 & 14.54 & 14.22 \\
${ }^{1} B_{2 u}$ & 19.58 & 18.69 & 18.67 \\
\hline
\end{tabular}

From Eqs. (21a) and (21b) in the text.

b From Eqs. (25a) and (25b) in the text.

- From Eqs. (31a), (31b), (32a), (32b) in the text. 
we may draw an analogy between the excited molecule and a set of interacting oscillators. Calculation of the lowest excitation energy of a given symmetry then corresponds to a search for the lowest mode of the set of coupled oscillators, which, as expected on classical grounds, occurs at a lower frequency than that of any one of the oscillators.

As Herzenberg et al. ${ }^{9}$ pointed out, the coupling together of many particle-hole excitations leads to a significant modification of the low-lying excited states of ethylene which can be interpreted in terms of a reduction in the effective interaction between the $\pi$ electrons by the dynamic polarization of the other electrons. This reduction is primarily a result of longrange, interbond, correlations as is evidenced by the fact that the dipole approximation for the Coulomb potential was sufficient to reproduce the significant features of this effect. They also estimated that there may be a collective state of ethylene at about $50 \mathrm{eV}$. No indication of such a state was found in this calculation (one excitation corresponding to an excitation energy of $32 \mathrm{eV}$ did have a heavy weighting in several elementary transitions; however, the oscillator strength was only 0.25 ).

On the basis of chemical intuition we would expect that many excitations would have a major component which essentially defines the excitation, thus allowing us to retain, in a limited sense, the classifications of excitations as being $\pi \rightarrow \pi^{*}$ etc. This is indeed the case for the lowest state of symmetry $B_{3 u}$ in ethylene, the $\pi \rightarrow \pi^{*}$ component comprising about $92 \%$ of the total wavefunction. However, the effect of the minor components of the excitation are far from being insignificant.

One of the assumptions of $\pi$-electron theory is that the $\sigma$ core is invariant to the disposition of the $\pi$ electrons, i.e., the $\sigma$ electrons are little affected by the precise form of the $\pi$-electron wave-function. However, the $\pi$ electrons are strongly coupled to the $\sigma$ electrons such that a rearrangement of one causes a corresponding rearrangement of the other. Allowing in some crude fashion for the polarization of the $\sigma$ core decreases the excitation energy by $\sim 15 \%$ and the oscillator strength by $\sim 30 \%$. At the same time the TDA results provide a theoretical justification for the success of the Pariser-Parr-Pople method. ${ }^{28}$ The net effect of the coupling between the particlehole pairs is to decrease the interaction between the $\pi$ electrons and justifies the use of empirical values for the matrix elements of $\pi$-electron theory smaller than the theoretical ones. By lowering the value of a certain integral, $\gamma_{p p}$, using spectroscopic arguments, Pariser ${ }^{29}$ achieved this required decrease in the electronic interaction. Thus, the downgrading of certain integrals is justified by molecular considerations as well as the

${ }^{28}$ R. G. Parr, Quantum Theory of Molecular Electronic Structure (W. A. Benjamin, Inc., New York, 1963), Chap. 3.

29 R. Pariser, J. Chem. Phys. 21, 568 (1953). proposed atomic considerations ${ }^{30}$ From the above we note that in a molecule, the $\pi$ electrons may be pictured as being immersed in a polarizable medium corresponding to the $\sigma$-electron density ${ }^{31}$ It might thus be possible to describe the effect of this polarizable medium on the $\pi$ electrons by means of a microscopic dielectric constant. Herzenberg et al. ${ }^{9}$ showed that within the set of approximations adopted by them, i.e., a dipole-dipole approximation for the Coulombic potential, that this indeed was the case-both the decrease in the excitation energy and the transition moment being explicable in terms of such a concept. This model can be rigorously justified by a selective summation of Feynman diagrams $^{32}$ or by a series of appropriate transformations on the Hamiltonian. ${ }^{33}$ Investigation of the feasibility of this approach for the study of the spectra of larger $\pi$-electron systems is presently under way.

In contrast to the $\pi$-electron calculations, we note that the methods presented here have no effect on the calculated ionization potential. The calculated value of $10.82 \mathrm{eV}$ is in excellent agreement with experiment (see Table I).

Also, of considerable interest is the application of the techniques presented here for the study of other molecules, e.g., acetylene, numerous diatomics, ${ }^{34}$ etc. At the present time, the set of calculations presented herein is being rerun using the Gaussian wavefunction for ethylene which was calculated by Moskowitz and Harrison. ${ }^{17}$ This will provide information on the sensitivity of the method to the accuracy of the ground-state wavefunction and, if the effects which have been shown to be operative here persist in the more detailed wavefunction, the results should provide a limit for the theoretical excitation energy and oscillator strength.

From a study of Table VIII in the last section, we see that in the STA four states lie below the ${ }^{1} B_{3 u}$ state while in the RPA no singlet state lies below it. ${ }^{35}$ This

${ }^{30}$ M. Orloff and O. Sinanoğlu, J. Chem. Phys. 43, 49 (1965). ${ }^{31}$ Contrary to the traditional viewpoint, the $\pi$ electrons are deeply immersed in the $\sigma$-electron density. See, for example, C. A. Coulson, N. H. March, and S. Altmann, Proc. Natl. Acad. Sci. (U.S.) 38, 372 (1952); M. D. Newton, F. P. Boer, and W. N. Lipscomb, J. Am. Chem. Soc., 88, 2367 (1966); A. C. Wahl, "Pictorial Studies of Molecules," Argonne National Lab. Tech. Rept. July, 1965. In these works it is shown that the $\sigma$-electron density exceeds that of the $\pi$ electrons throughout most of the bonding region, including the region in which the $\pi$-electron density is greatest.

${ }_{32}$ D. Falkoff, in Lecture Notes on the Many-Body Problem from the First Bergen International School of Physics-1961 (W. A. Benjamin, Inc., New York, 1962).

${ }_{33} \mathrm{R}$. Harris (private communication).

${ }^{34}$ For application of the STA see C. W. Sherr, J. Chem. Phys. 23, 569 (1955); $\mathrm{N}_{2} ; \mathrm{J}$. W. Richardson, ibid. 35, 1829 (1961), $\mathrm{N}_{2}$; H. Brion and C. Moser, ibid. 32, 1194 (1960); CO; B. J. Ransil, ibid. 35, 669 (1961); $\mathrm{F}_{2}, \mathrm{HF}, \mathrm{Li}_{2}, \mathrm{LiH}, \mathrm{N}_{2}$, and others; and $\mathrm{R} . \mathrm{K}$. Nesbet, ibid. 43, 4403 (1966); $\mathrm{N}_{2}$, CO, BF. For applications of the TDA see H. Lefebvre-Brion, C. Moser, and R. K. Nesbet, ibid. 35, 1702 (1961); CO.

${ }^{35}$ Note that this is in disagreement with the CI results of $R$. Polak and J. Paldus, Theoret. Chim. Acta 5, 422 (1966), who obtained a reversal of the energy levels and found an excited singlet state $\left({ }^{1} B_{2 g}\right)$ much below ${ }^{1} B_{3 u}$. However, their use of the Mulliken approximation to evaluate the multicenter integrals negates much of the quantitative significance of the calculation. 
is in contrast to the results of Robin et al..$^{36}$ who, with a set of Guassians augmented with expanded orbital exponents, find two states below ${ }^{1} B_{3 u}$, namely, ${ }^{1} B_{1 u}$ and ${ }^{3} B_{1 u}$. In our calculations the four states lying closest to the ${ }^{1} B_{3 u}$ state are of symmetry $B_{3 g}$ and $B_{2 g}$, the former corresponding to the Berry ${ }^{37}$ assignment of the mystery band of ethylene. The state proposed by Robin $e t a l$. for the mystery band, $B_{1 u}$, is found to lie at rather high energies, $\sim 14 \mathrm{eV}$, even in the RPA. Thus, our calculations cannot account for a mystery band lying below the ${ }^{1} B_{3 u}$ state. To prevent misinter-

${ }^{36}$ M. B. Robin, R. R. Hart, and N. A. Kuebler, J. Chem. Phys. 44,1803 (1966).

${ }^{37}$ R. S. Berry, J. Chem. Phys. 38, 1934 (1963). pretation, however, it should be pointed out that because of the nature of the ground-state calculation (i.e., the use of a minimum basis set), the above results cannot provide a definitive answer to the question of the mystery band of ethylene.

\section{ACKNOWLEDGMENTS}

We wish to thank Dr. W. E. Palke and Professor W. N. Lipscomb for communication of results prior to publications and to acknowledge the use of the matrix diagonalization routine of Mr. S. Persselin. One of us (T.H.D.) thanks the Woodrow Wilson Foundation for fellowship support.

\title{
Vibrational Spectra of Molten Salts. II. Infrared Spectra of Some Divalent Metal Nitrates in Alkali-Metal Nitrate Solutions*
}

\author{
R. E. HESTER AND K. KRISHNAN \\ Chemistry Department, University of York, York, England
}

(Received 27 March 1967)

\begin{abstract}
Infrared spectra of thin films of $\mathrm{Mg}\left(\mathrm{NO}_{3}\right)_{2}, \mathrm{Sr}\left(\mathrm{NO}_{3}\right)_{2}, \mathrm{Ba}\left(\mathrm{NO}_{3}\right)_{2}, \mathrm{Cd}\left(\mathrm{NO}_{3}\right)_{2}$, and $\mathrm{Pb}\left(\mathrm{NO}_{3}\right)_{2}$, in molten $\mathrm{KNO}_{3}$ and equimolar $\mathrm{KNO}_{3}-\mathrm{NaNO}_{3}$ mixtures have been obtained over the spect ral region $4000-200 \mathrm{~cm}^{-1}$. All the nitrates except those of $\mathrm{Mg}$ and $\mathrm{Cd}$ were in the anhydrous state. Even at temperatures of $300^{\circ} \mathrm{C}$ or more, the magnesium and cadmium nitrate melts retained small amounts of water. As the concentrations of the divalent metal nitrates were increased, some nitrate bands split, and new low-frequency lines were observed in some cases. These are located at 380 and $340 \mathrm{~cm}^{-1}$ for $\mathrm{Mg}\left(\mathrm{NO}_{3}\right)_{2}$ solutions, at $520 \mathrm{~cm}^{-1}$ for $\mathrm{Cd}\left(\mathrm{NO}_{3}\right)_{2}$, and around $420 \mathrm{~cm}^{-1}$ for $\mathrm{Pb}\left(\mathrm{NO}_{3}\right)_{2}$. The splitting of the $\nu_{3}$ line of free nitrate decreases in the order $\mathrm{Mg}^{2+}>\mathrm{Cd}^{2+}>\mathrm{Ca}^{2+}>\mathrm{Pb}^{2+}>\mathrm{Sr}^{2+}>\mathrm{Ba}^{2+}$. Except for $\mathrm{Sr}^{2+}$, this is the order of the ionic potentials. The observed bands are assigned and discussed in terms of complexes formed between metal and nitrate ions. Relative strengths of nitrate and chloride binding by $\mathrm{Cd}^{2+}$ and $\mathrm{Mg}^{2+}$ ions have been investigated by addition of $\mathrm{Cl}^{-}$ion to the mixed nitrate systems.
\end{abstract}

\section{INTRODUCTION}

Previous work ${ }^{1}$ has indicated that detailed information on the structures of complex species formed in molten salt systems can be obtained through a study of their infrared absorption spectra. Our earlier work showed definite calcium-nitrate complexes to exist in molten $\mathrm{Ca}\left(\mathrm{NO}_{3}\right)_{2} / \mathrm{KNO}_{3} / \mathrm{NaNO}_{3}$ systems. We were able to determine the magnitude of the perturbation of the nitrate ion in these systems, and to show that the complex formation equilibria resembled quite closely those occurring in aqueous solution. We have now extended this work to a much wider range of divalent metal nitrates in order to establish a more general understanding of molten salt solutions of this kind.

A Raman study has been made ${ }^{2}$ of molten mixtures of all the alkaline-earth metal nitrates in $\mathrm{NaNO}_{3}$, and of

\footnotetext{
* This study was aided by a grant from the Office of Saline Water, U.S. Department of the Interior.

${ }^{1}$ R. E. Hester and K. Krishnan, J. Chem. Phys. 46, 3407 (1967).

${ }^{2}$ G. J. Janz and T. R. Kozlowski, J. Chem. Phys. 40, 1699 (1964).
}

some of them in $\mathrm{KNO}_{3}$ as a solvent. We have effectively repeated this vibrational study, but have used the infrared region as our probe, and have extended the investigation to systems involving $\mathrm{Cd}$ and $\mathrm{Pb}$ nitrates. In addition to being able to study low-frequency fundamentals of the free and complexed nitrate ion, the infrared spectrum provides considerable additional information in the form of strong overtone and combination bands. The different selection rules for the appearance in infrared and Raman spectra of the vibrational fundamentals of $\mathrm{NO}_{3}^{-}$ions having $D_{3 h}$ symmetry allows us to observe the out-of-plane vibrational frequency, though this is inactive in the Raman spectrum. Though the in-plane symmetric breathing mode of the $\mathrm{NO}_{3}{ }^{-}$ion is formally forbidden in the infrared, in practice it is usually observed, even in the absence of further signs of nitrate perturbation, in such systems as pure alkali-metal nitrate melts, ${ }^{3}$ or even in aqueous alkalimetal nitrate solutions. ${ }^{4}$

${ }^{8} \mathrm{~J}$. K. Wilmshurst and S. Senderoff, J. Chem. Phys. 35, 1078 (1961).

${ }^{4}$ D. E. Irish and G. E. Walrafen, J. Chem. Phys. 46, 378 (1967). 